\title{
A GIS-BASED MULTICRITERIA SPATIAL DECISION SUPPORT SYSTEM MODEL TO HANDLE HEALTH FACILITIES RESOURCES. CASE OF CRISIS MANAGEMENT IN BATNA, ALGERIA
}

\author{
Belkacem LAHMAR ${ }^{1}$, Hadda DRIDI ${ }^{2}$, and Ahmed AKAKBA ${ }^{3}$
}

DOI: $10.21163 / G T \_2020.151 .16$

\begin{abstract}
:
The city of Batna is exposed to risks of different natures and varied occurrences and cannot be avoided, the available resources to face up the results of catastrophes become very limited, especially the healthcare system, that must be flexible and take care all wounded fast as possible but also it should take in consideration their capacity and managing their resources, but it suffers from structural problems which has a negative effect on its resilience that help to rebound and assume to the normal functioning after a disaster. This paper aims is to develop a spatial model and present the possibility of the implementation inside GIS (Geographic Information System) environment to handle the problem of health resources (workforces and medical supply), and evaluate the evacuation plane in crisis management by find and the closest health centre that can take care the wounded within an acceptable distance, and in the same time the model should chose the center with available capacity.
\end{abstract}

Key-words: Model, health services, crisis management, Capacity.

\section{INTRODUCTION}

The prevention of major risks represents one of four principal phases in PPRR model (Fig. 1), and the management of disasters within the framework of sustainable development constitute a global system initiated and led by the State, implemented by public institutions and local authorities.

Like many counties, Algeria is ranked height vulnerable to risks, and as result every few years one the major risks cause huge human losses and damages in infrastructure, economic and materials; specially risks related to:

- earthquakes and geological risks,

- flooding, climate risks,

- forest fires,

- industrial and energy risks,

- risks related to human health.

Historically Algeria knows number of disasters most of them are naturals but with significant losses and economic damages. The Table 1 (Disaster history 1962-2012 in Algeria) shows some of them.

1 University of Batna 2, Laboratory of Natural Hazards and Spatial Planning (LRNAT), Batna, Algeria,b.lahmar@univ-batna2.dz/Belkacemlahmer05@gmail.com, ORCID ID:0000-00028479-8982 Tel:(+213)657221097.

2 University of Batna 2, Laboratory of Natural Hazards and Spatial Planning (LRNAT), Batna, Algeria, Hadda.dridi@gmail.com

3 University of Batna 2, Laboratory of Natural Hazards and Spatial Planning (LRNAT), Batna, Algeria,dja_aka@yahoo.fr 


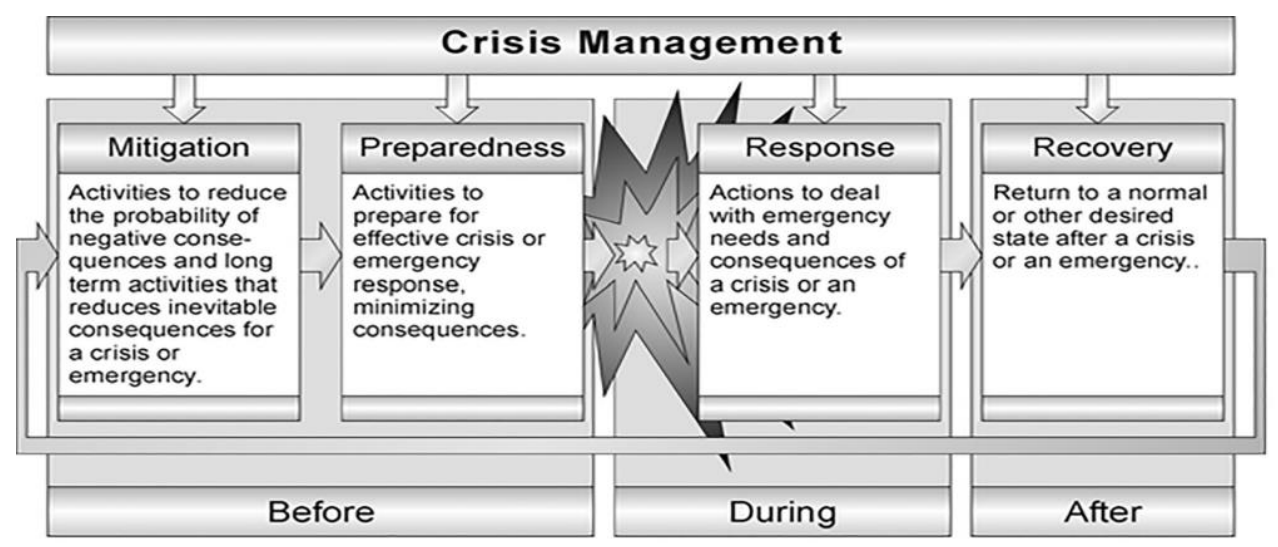

Fig. 1. Crisis management model (PPRR). Source (Rogers and others 2011).

To avoid this problem, Algeria created a disaster management system, this program consists of emergency planning and responses which is relief planning called the ORSEC plan and special intervention planning. ORSEC is a territory plan and every city's local government assure execution of this plan in their territory during of major risks to ensure tents, chalets, or any other means of temporary accommodation, food and clean water, emergency medical; it composed of multiple modules to support and manage every particular aspect of a disaster, and must identify the following priorities:

- Rescue of people.

- The establishment of secure temporary hosting sites.

- The safety and health of the victims and their property.

- The establishment of energy supply.

- Internal intervention plans.

- Special intervention plans.

Table 1.

Disasters history 1962-2012 in Algeria. Source (protection 2017).

\begin{tabular}{|l|l|l|l|}
\hline \multicolumn{1}{|c|}{ Date } & Location name & \multicolumn{1}{c|}{ Type of risks } & \multicolumn{1}{c|}{ Damages } \\
\hline 10.10 .1980 & Chlef & Earthquake & $\begin{array}{l}\text { 2633 deaths, 8369 injured,70\\
% of building } \\
\text { destroyed }\end{array}$ \\
\hline 18.08 .1983 & Jijel & Forest fire & $\begin{array}{l}\text { 11 deaths,19 burned person, burned area of } \\
27000 \text { hectare }\end{array}$ \\
\hline 10.11 .2001 & Bab El-Oued & Flood & 900 deaths \\
\hline 21.05 .2003 & Boumerdess & Earthquake & $\begin{array}{l}2278 \text { deaths,11000 injured,18000 without } \\
\text { shelter }\end{array}$ \\
\hline 11.08 .2008 & Ghelizan & Gaz explosion & 21 deaths \\
\hline 22.02 .2012 & El-Taref & Flood & 2 deaths, significant material damage \\
\hline
\end{tabular}

One of the most important ORSEC module is the health module, but this last comprises several structural problems, such as random health facility distribution to population distribution, financing problems and difficulty adapting to new challenges which reflected in their functions (Chachoua 2014; Bouyoucef barr 2015), despite the decentralization of the national health system (SNS) and the dualism of health (Oufriha 2006), for better adaptation to local issues and reaction speed against local diseases and in case of significant risks; in addition, more than $48 \%$ of patients don't respect the graduation of care even in the 
case of very simple ailments that do not require hospital care (Nasereddine 2016), so there is a random of distribution of patient, and the hospital capacity is overload and rich its maximum capacity, this situation decrease its efficiency and healthcare quality. Also, the health system in Algeria is not updated to adapt new spatial planning technologies like GIS.

The aim of this paper is to create a model for crisis management and implement in GIS environment, this model will deal with health module emergency needs and manages their capacity (workforces, medical supply...etc) in the response phase of crisis management model, in the same time it will assure the evacuate plane of wounded during a crisis to the closest health facility that has the ability to handle them.

\section{STUDY AREA}

The city of Batna is strategically located in eastern Algeria. Its geographical location represents an important crossroads connecting the east, west, north, and south of the country. Batna has experienced since its foundation (1844) a strong but random urban dynamic in terms of demography and space, which results a functional imbalance in public services. This affects the health service quality of over 301,708 inhabitants who live in the city (DSPB 2017). The city is located in a high-temperature zone $\left(+35 \mathrm{C}^{\circ}\right.$ in summer) and moderate precipitation and low slope (3\%-5\%), these parameters increase the vulnerability of transmission epidemic problems whereas (Bendib et al. 2016; Issam and Said 2017) indicates that $48.89 \%$ of the total area of Batna is highly vulnerable to risks related to human health. To avoid this problem the local health department creates fourteen of health facilities to ensure primary healthcare and focus on optimizes the public health in the city and increase the accessibility to health care services (DSP 2017) (Fig. 2).

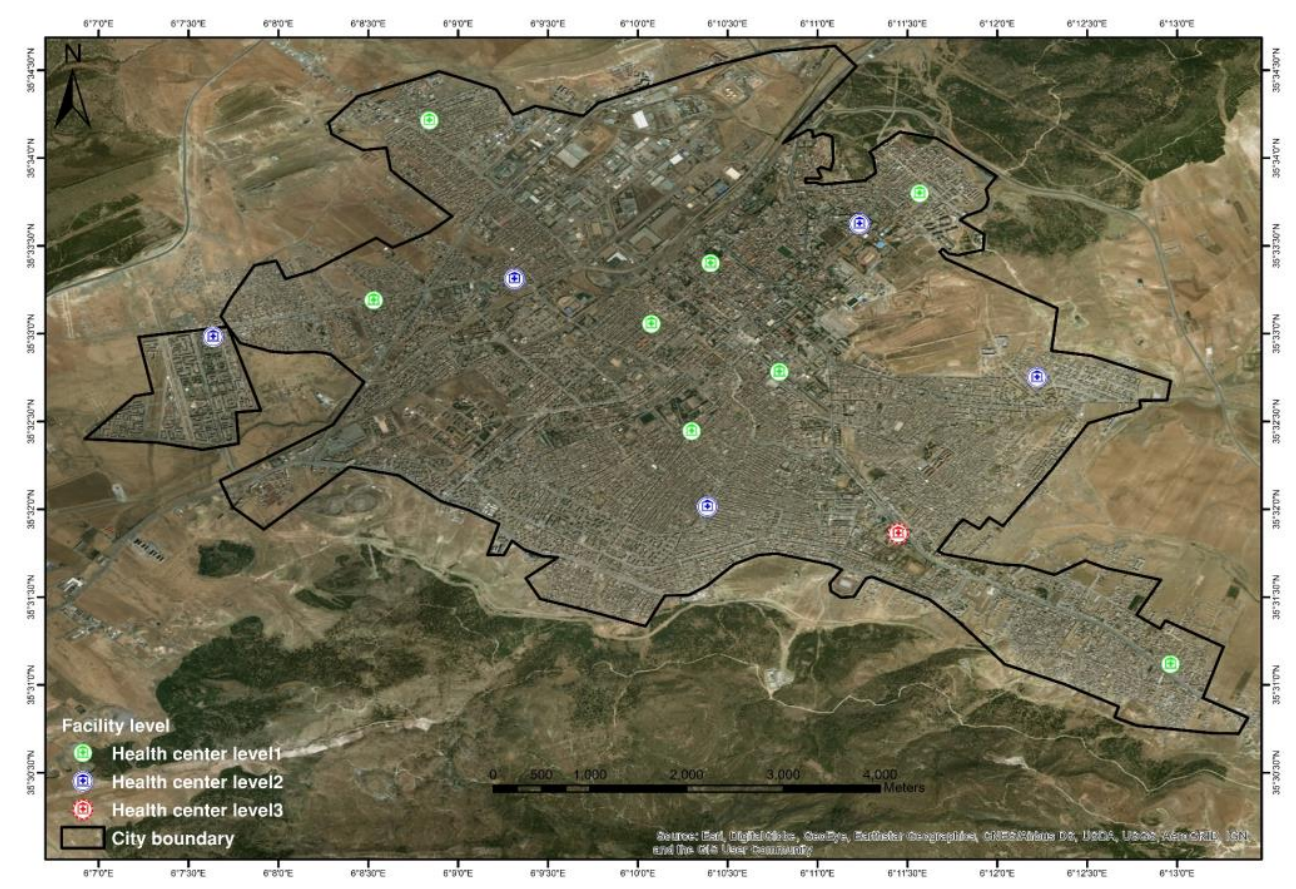

Fig. 2. Study area and health facilities distribution. 


\section{METHOD AND APPROACH}

\subsection{GIS in healthcare and crisis management}

A GIS is a framework for gathering, managing, and analysing data, rooted in the science of geography, it integrates many types of data. It analyses spatial location and organizes layers of information into visualizations using maps (ESRI 2008). This unique capability helping user to make smarter decisions. It provides a strong and dynamic technique that allows us to model, manage geographic relationships, make a better decision, improve functional performance, generate more cost-effective citizen plans, evaluate spatial details, and modify information and maps (Meika 2010) .

In Addition, GIS push forward the health geographer to use range methods (quantitative, qualitative, spatial analysis) with answer the who gets what, where and why with respect to illness and appropriate care (Cromley and McLafferty 2011), also it can provide answers to the following questions, such as: How many paramedic units are required and where should they be located? What evacuation routes should be selected? does the storage facility is sufficient? Will the road networks handle the traffic? What quantity of supplies, bed space, and so forth (Johnson 2000), also it's a helpful tool for decision makers and they would enable them to make the appropriate decisions in the real time? There are also various functions which GIS can provide in the response missions such as: prepare maps, briefs, and status reports (ESRI 2008).

\subsection{Spatial Decision Support Systems (SDSS)}

SDSS has grown dramatically over the last few decades but there is still no universally accepted definition (Sugumaran and Degroote 2010), however we choose the definition of (Malczewski 1999) "The Spatial Decision Support Systems is an interactive computer based system designed to support a user or group of users in achieving a higher effectiveness of decision making while solving a semi-structured spatial decision problem". So basically, it's the application of Decision Support Systems (DSS) to solve problems and find the best actions (solutions) to move from an initial situation to a desired goal situation where the spatial and the geography in general are important (Fig. 3).

SDSS is technique executes mostly in GIS environment and widely in emergencies (Cova 1999; Gunes and Kovel 2000), but this classic operation of crisis management is focus on geographical part like scenario of evacuation and choose best street path in emergency situations (Nicoară and Haidu 2014; FILALI and KALLA 2016; Cadar et al. 2017; HASNAT et al. 2018), or focus on supply chain (medical supply, workforces) and the capacity of health (bed, The operating rooms...etc), but SDSS is a combined analytical tools with GIS environment and ensure multi criteria analyses, iterate the problem solving, have interactive user interface, offer the report generation and visualization, spatial analysis and spatial modelling capability (Mora et al. 2003; Sugumaran and Degroote 2010) (Fig. 3), so it represents a platform where the crisis management is done by taking the advantage of spatial data management and analysis, interactive user interface and semi or ill-structured problem solving for better decision in real time. 


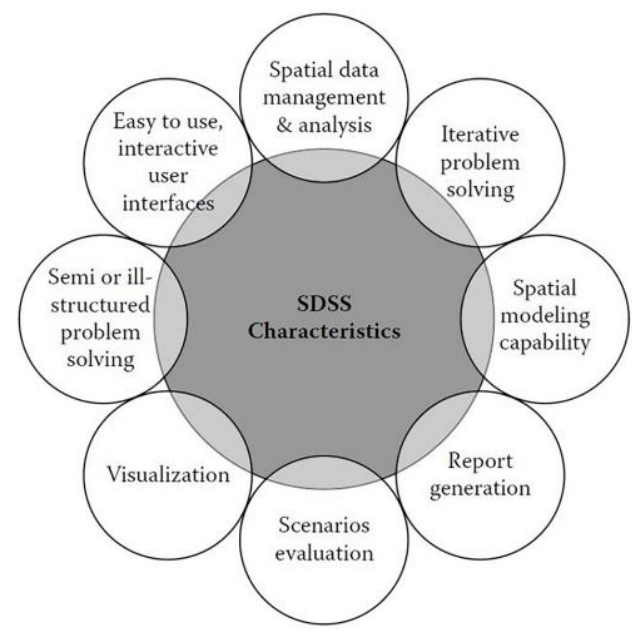

a)-Characteristics of SDSS

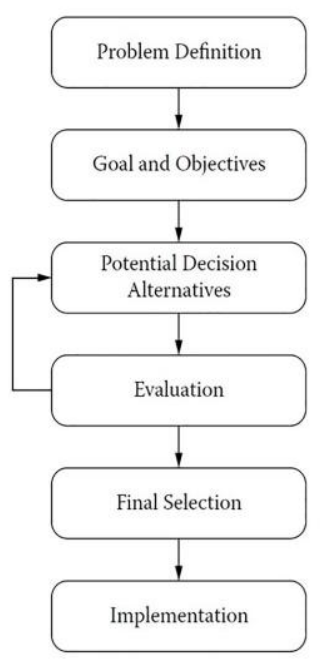

b)-General spatial decision-making process

Fig. 3. Characteristics of SDSS.

SDSS has several utilization combined with GIS and database management system (DMS) in different domain whether urban and planning field, agriculture or in housing (Coutinho-Rodrigues et al. 2011; Sultani et al. 2009; Makropoulos et al. 2003; Ayeni 2003; Zhang et al. 2009; Ascough et al. 2002) or in logistics and transportation domain (RUDA 2014); and it's a main part as well in risks and health (Wangdi et al. 2016). Even government agencies uses this technique like Hazus ([n.d.]) which is multi-hazard analysis software suite for the US Federal Emergency Management Agency (Schneider and Schauer 2006), CommunityViz is also a SDSS extension for ESRI ArcGIS, it offers the ability of analysis tool used for, among other applications, urban planning, land use planning, geodesign, transportation planning and resource management applications (2003). The SDSS is not just for crisis and urban management but it can be used for various domain like iGLASS which an open source SDSS for public school location-allocation (Chen et al. 2018).

\subsection{Health facilities data}

The city of Batna counts a total of fourteen functional health centre divided into three categories (DSP 2017):

(1) Treatment room: represent the lowest level in the (SNS), it offer basic health services only, with a low number of physicians and their availability is limited for 8 hours per day, it's for small area geographically and to serve a low number of population (refers to health facility from the level 1 in our paper).We count eight treatment room in the city.

(2) Multi-service clinic: represent the 2 nd level, it offers some advanced services with a higher number of physicians with and resources like dentists and biological analyst compared to the treatment room, availability services is 12 hours per day and in some cases 24/24 hours, In Batna we have five health facility from this level.

(3) Hospital: The city has one hospital, it includes number of services such emergency service, it offers a 24/24 hours and 7/7 days services and has a large geographically cover all the state of Batna, it represents the 3rd level. 


\subsection{The capacity}

As capacity can sometimes change over time, it should be assessed at the time that consent is required. This will usually be done by an appropriately trained and experienced healthcare professional who's either: recommending the treatment or investigation, involved in carrying it out. The capacity may refer to medical, or any resources that can be an obstacle to health care service. In our case the capacity refers to available medical staff who will caring the wounded.

\subsection{Software and tools}

The tools used is ModelBuilder which is a powerful graphical interface allows us to use different tools, variable to create models inside ArcGIS environment. The models are workflows that string together sequences of geoprocessing tools, feeding the output of one tool into another tool as input. ModelBuilder can also be thought of as a visual programming language for building workflows. While ModelBuilder is very useful for constructing and executing simple workflows, it also provides advanced methods for extending ArcGIS functionality by allowing you to create and share your models as tool (Institute 2018).

\section{RESULT}

\subsection{Spatial health model for crisis management}

The proposed model is organized into layers and every layer has an input part, a processing part and an output (Fig. 4), so every layer has a specific task like below:

(1) Layer 1: the input part (input 1) initialize all required parameters in the model and start the first step of the processing:

- Variables in layer 1:

○ Field_FacilityLayer: The attribute field of the health facility level. condition.

- Field_Catastrophe_Cas: The attribute field of the wounded health

○ Facility level: The health centre feature layer.

- Catastrophe: result victims and wounded feature layer.

- The Processing in layer 1: it has four sub processing tools:

○ 'Iterate Field value': the first order process is iterating through all values of the wounded health condition in catastrophe feature layer.

○ 'Select Facility Layer by Attribute': get all health facility feature that match the value from Iterate Field value process.

○ 'Select Catastrophe Layer by Attribute': find all catastrophes feature that match the value from Iterate Field value process.

$\circ \quad$ 'Calculate Value': prepare a name to assign it to solved route in layer 3.

(2) Layer 2: The objective of this layer is to find the closest health facility that match the casualty level in the capacity. The input part (input 2) is the output of the first layer as explained in the follow:

- Variables in layer 2: results.

○ 'FDB.GDB': the geodatabase location path where we will store the final 
○ 'X Barriers parameters': barriers are feature classes in network analysis layers that restrict or alter costs of the underlying edges and junctions of the associated network dataset.

○ 'Dataset': the network dataset to execute the network analysis tool.

○ 'Catastrophe_Filter' and 'Facility_Filter': the outputs of the first layer, represent a selected feature from previous layer (Layer 1).

- The Processing in layer 2: The processing part in this layer is 'Find Closest Facility' which find the closest facility (evacuation path) based on the street map of the city.

(3) Layer 3 and 4: are simply update the capacity of the chosen health centre.

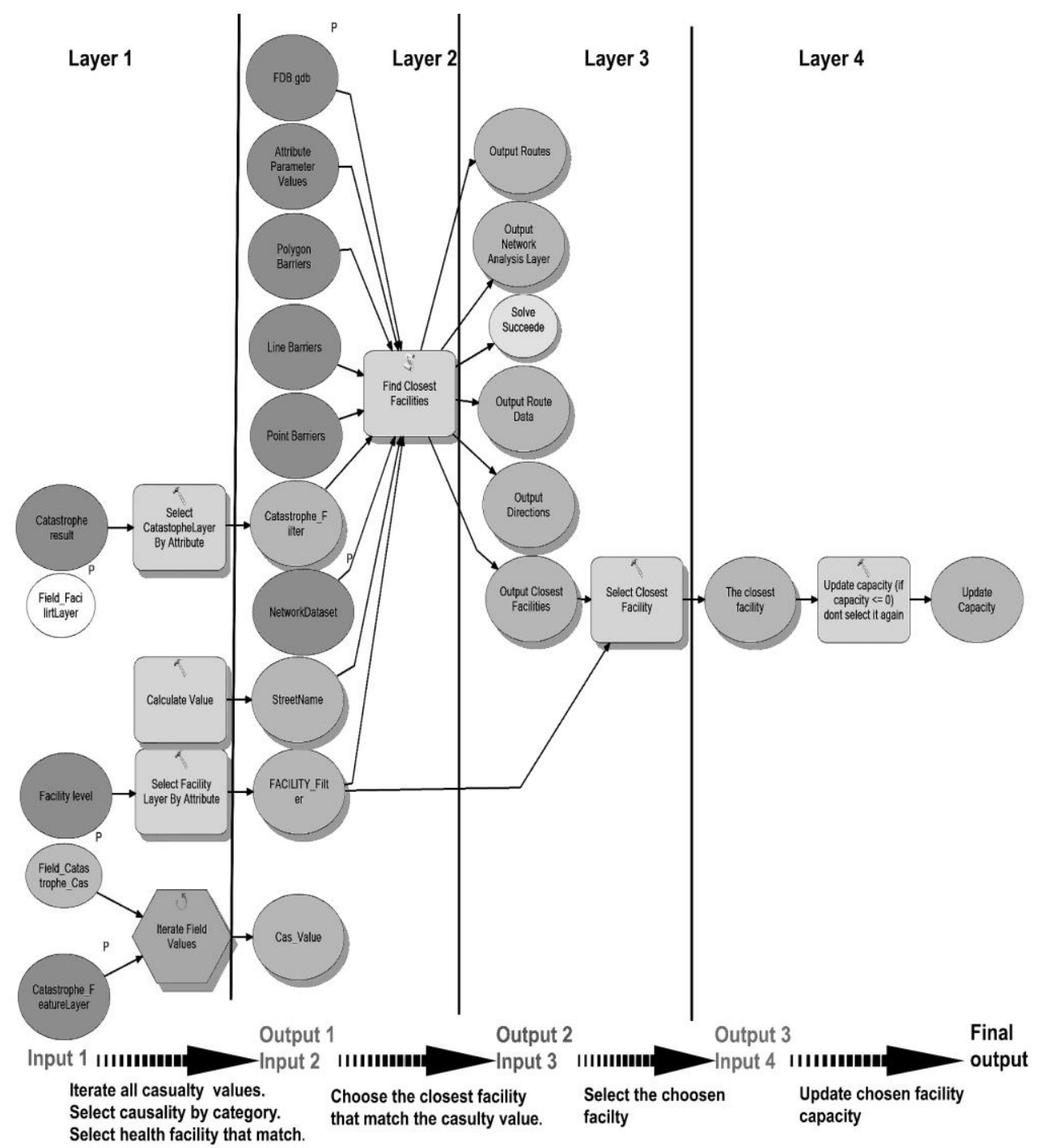

Fig. 4. Spatial health model for crisis management. 


\subsection{Spatial health model for crisis management python code}

Since the ModelBuilder uses python as the main programming language, the python code of Spatial health model for crisis management was generated with respect to the python syntax and format like the following: green colour represent comments, blue represent the reserved keywords in python, red is the argument and parameters and black colour represent the variable name (See the Model.py code file at supplement material).

\# Import arcpy module

import arcpy

\# Load required toolboxes

arcpy.ImportToolbox("Model Functions")

\# Script arguments

Field_Catastrophe_Cas $=$ arcpy.GetParameterAsText $(0)$

if Field_Catastrophe_Cas $==$ ' 'स' or not Field_Catastrophe_Cas:

Field_Catastrophe_Cas = "Cas" \# provide a default value if unspecified

Catastrophe_FeatureLayer $=$ arcpy.GetParameterAsText $(1)$

if Catastrophe_FeatureLayer $==$ '\#' or not Catastrophe_FeatureLayer:

Catastrophe_FeatureLayer = "FDB.gdb $\backslash$ Catastrophe" \# provide a default value if unspecified

Field_FacilirtLayer $=$ arcpy.GetParameterAsText $(2)$

Dataset $=$ arcpy.GetParameterAsText $(3)$

if Dataset $==$ '\#' or not Dataset:

Dataset $=$ "Dataset" \# provide a default value if unspecified

FDB_gdb $=$ arcpy.GetParameterAsText(4)

if FDB_gdb == '\#' or not FDB_gdb:

FDB_gdb = "FDB.gdb" \# provide a default value if unspecified

This first part initiates the environment and load necessary package 'arcpy' that contain the main core functionalities, after import 'arcpy' package the declaration of arguments and data location and default values for every parameter is the second step.

\# Local variables:

Cas_Value $=$ Catastrophe_FeatureLayer

Catastrophe_result $=$ "Catastrophe result"

Catastrophe_Filter $=$ Catastrophe_result

Facility_level = "Facility level"

FACILITY_Filter $=$ Facility_level

The_closest_facility $=$ FACILITY_Filter

StreetName = "Cas3"

Point_Barriers = "in_memory $\backslash\{$ B62375D9-0E03-4E3A-AB40-DD1EECA92C7E $\}$ "

Output_Routes = Point_Barriers

Output_Directions $=$ Point_Barriers

Output_Closest_Facilities $=$ Point_Barriers

Line_Barriers = "in_memory $\backslash\{$ ED87C092-03B8-4616-83B7-E1D7AEBB244C $\}$ "

Polygon_Barriers = "in_memoryl\\{803582EC-323C-478F-954B-35E590976E40 $\}$ "

Attribute_Parameter_Values = "in_memorylI\{1EE55C2C-CDD3-4B4B-8B47-A376215B4871\}"

Solve_Succeeded = "true"

Output_Network_Analysis_Layer = ""

Output_Route_Data = ""

Update_Capacity $=$ The_closest_facility 
The second part declares all local variables used in the spatial health model for crisis management, and assignment value to every variable. There is three variable type:

- A feature variable: represent a vector layer in the geodatabase, stored in the hard drive.

- In memory variables: they are feature layer stored in memory because the model needs them temporary but not stored in the hard drive.

- Constant and real time variable: the values of this type assigned in real time (dynamic) or as constant value before executing the code.

\section{\# Process: Iterate Field Values}

arcpy.IterateFieldValues_mb(Catastrophe_FeatureLayer, Field_Catastrophe_Cas, "String", "true", "false", "')

Iterate field values part using 'IteratorFieldValues_mb' tool which iterate over each value in a given field (Iterate Field Values-Tools | Documentation), the first parameter is 'Catastrophe_FeatureLayer', represent the data table that will be iterated over, the second parameter is the field name for iteration, since the model will uses the injured case to choose the best health facility, the injured case is stored in 'Field_Catastrophe_Cas' field, the third parameter is 'String' which represent a text represent the data type of the output value.

Since we want get the type of injured, we choose 'true' for the fourth parameter that represent a 'boolean' value to determine if the iteration values will be based on the unique value of the specified field or will run for each record in the input table, The fifth and last parameters determines if null values in the field will be skipped or not, and specify the null value to skip.

\# Process: Select CatastopheLayer By Attribute

arcpy.SelectLayerByAttribute_management(Catastrophe_result, "NEW_SELECTION", "\%Field_Catastrophe_Cas\%='\%Cas_Value\%"')

\section{\# Process: Select Facility Layer By Attribute}

arcpy.SelectLayerByAttribute_management(Facility_level, "NEW_SELECTION", "Level = '\%Cas_Value\%' AND Capacity $>0$ ")

Select the injured by attribute that match the 'Cas_Value'. The 'cas_Value' represent the injured case that selected from previous code, 'Field_Catastrophe_Cas' represent the field that contain information about injured. Every case is grouped into new selection that contain the case that a facility can accept and has the ability to take care.

The same thing for health facilities, the select layer by attribute selects the health facilities that candle the selected case and in the same has a capacity above 0 so as example if we get 'value1' from previous code then the selection of facility will be all facilities of level = value 1 and the selection of injured will be Fielcatatropheccas $=$ value 1 and so on. 
\# Process: Calculate Value

arcpy.CalculateValue_management("'\%Cas_Value\%"', "', "String")

\section{\# Process: Find Closest Facilities}

arcpy.FindClosestFacilities_na(Catastrophe_Filter, FACILITY_Filter, "Meters", Dataset, FDB_gdb, StreetName, "D3", "D4", "1", "', "TRAVEL_TO", "', "NOT_USED", "GEO_LOCAL",

"ALLOW_UTURNS", Point_Barriers, Line_Barriers, Polygon_Barriers, "Length_2", "Seconds", "Length", "Meters", "NO_HIERARCHY", "', Attribute_Parameter_Values, "', "20 Kilometers", "I"g_osm_pt" \#; |"g_osm_lnl" \#;|"g_ND_Junctionsl" \#", "TRUE_LINES_WITHOUT_MEASURES", "10 Meters", "DIRECTIONS", "en", "Miles", "NA Desktop", "', "', "'", "', " ", "', "'", "NO_SAVE_OUTPUT_LAYER", "CUSTOM", "', "NO_SAVE_ROUTE_DATA")

This part is simply finding the closest facility to injured person locations. The health facilities layer is the result of 'selectlayerbyattribute' from previous code and the same thing for injured.

\# Process: Select Closest Facility

arcpy.SelectLayerByLocation_management(FACILITY_Filter, "ARE_IDENTICAL_TO", Output_Closest_Facilities, "', "NEW_SELECTION", "NOT_INVERT")

\# Process: Update capacity (if capacity $<=0$ ) dont select it again

arcpy.CalculateField_management(The_closest_facility, "Capacity", "[Capacity]= [Capacity]-1", "VB", "')

The final part is updating the capacity of selected health facility. This done on two steps: select the closest facility, then use 'CalculateField_management' to update field capacity of the selected health facility.

\subsection{Mapping the evacuation plane}

The objective of the model is to manage the health module in response phase during crisis management geographically and the capacity and resources, the model should fit the following objectives:

- select all facilities with capacity and resources as a first step,

- the second step is finding the closest facility to injured,

- in the last update the capacity and resources immediately.

- Detect the health centres with capacity and level the handle every injured condition.

To test the proposed model, we simulate a risk that result 70 victims with different health conditions and we execute the model to check if the model can find the closest facilities (with good capacity). In the 2nd step we execute the model again just after the first execution, because after evacuate the injured persons in first execution the capacity of the selected health centres has been updated (Fig. 5). 
A- The first execution.

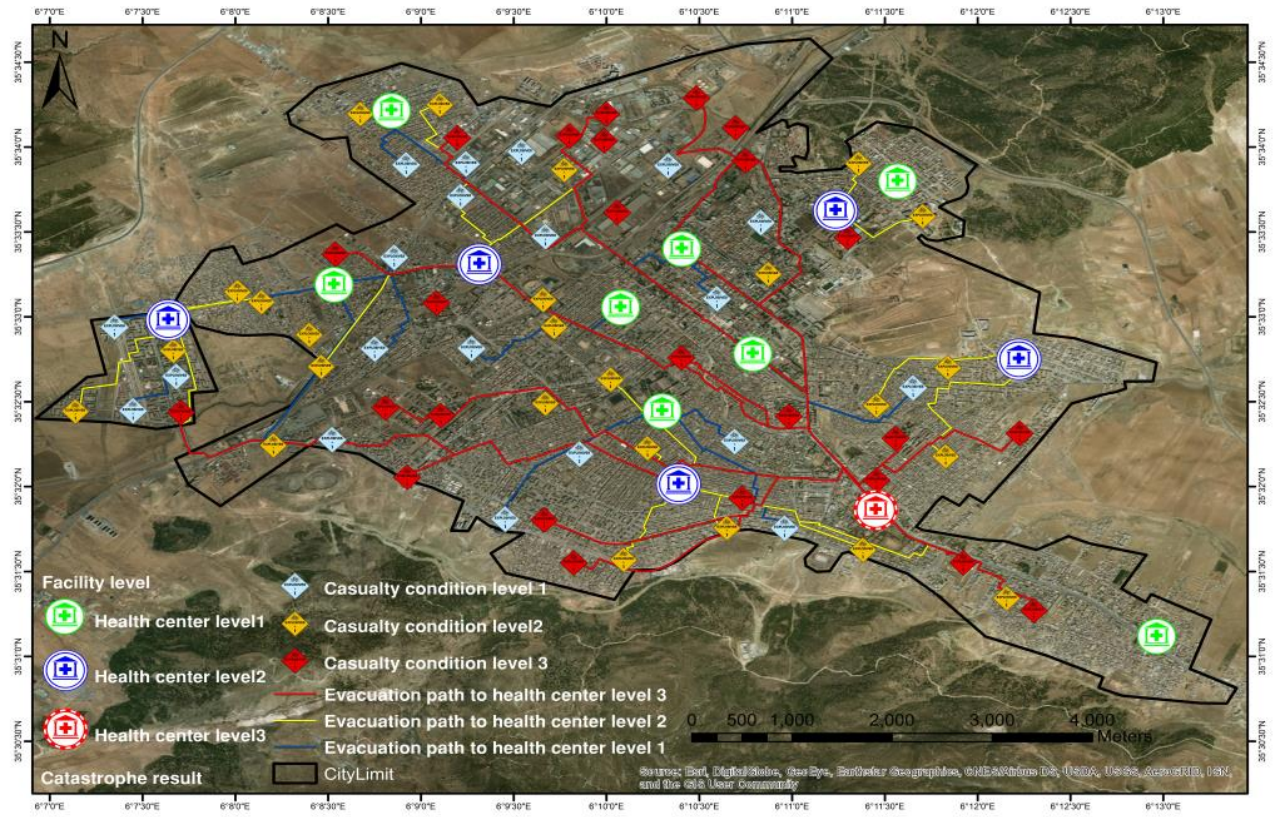

\section{B - The second execution.}

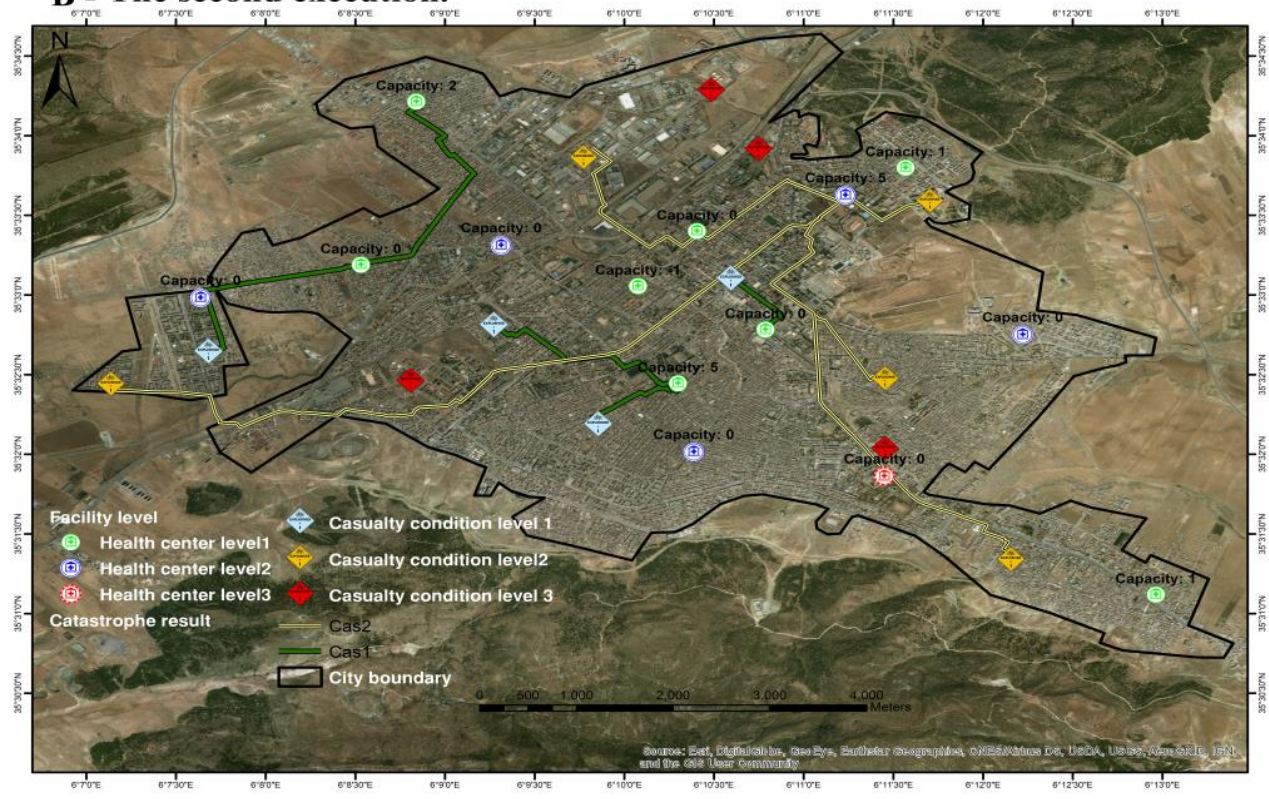

Fig. 5. Result of testing Spatial health model for crisis management. 


\section{DISCUSSION}

The crisis management model shows very interesting results in the side of management of risks result and evacuation plane, and it's clearly fit our objective and could be using in crisis management cell and it's offered the following possibilities:

- management the data flow problem in real time.

- The geographical distribution of wounded and victims between available health centres, which mean increase the effectiveness of the evacuation plane and the health system in the city.

- Avoid the overload problem since the city has only one hospital.

- Managing data capacity in the health centres in real time so we can detect any overload or low capacity, medical supply or workforces.

- $\quad$ in case of any health centre is reached its maximum capacity, the management cellule crisis can reinforce it by send workforces.

After testing the crisis management model, we detect that the health system of Batna city is weak and is not effective, since evacuate 70 victims in the same time overload all health centres and they reached maximum capacity by $100 \%$, and in case of significant risk the city need reinforcement from outside the city.

\section{ACKNLOWGMENT}

I would like to express my gratitude to Pr. KALLA Mahdi director of the LRNAT lab in university of Batna 2 who guided me throughout this project, and Dr. GUELLOUH Sami who helped me in collecting health data.

\section{R E F E R E N C E S}

Ascough, J., Rector, H., Hoag, D., McMaster, G., Vandenberg, B., Shaffer, M., ... Ahjua, L. (2002). Multicriteria spatial decision support systems for agriculture: Overview, applications, and future research directions. Integrated Assessment and Decision Support Proceedings of the 1st Biennial Meeting of the IEMSS. U.S. DEPARTMENT OF AGRICULTURE: Agricultural Research Service, pp. 175-180.

Ayeni, B. (2003). The design of spatial decision support systems in urban and regional planning. Decision Support Systems in Urban Planning. London: Routledge, pp. 20-33.

Bendib, A., Dridi, H., Kalla, M. and Baziz, N. (2016). Spatial analysis of typhoid fever vulnerability in the city of Batna (eastern Algeria). Environnement, Risques \& Santé, 15(3), 228-237.

Bouyoucef barr, D. (2015). L'organisation territoriale de l'offre de soins : quelles réalités en Algérie. La Revue des Sciences Commerciales, 14(1), 70-85.

Cadar, R.D., Boitor, M.R. and Dumitrescu, M. (2017). Effects of traffic volumes on accidents: The case of Romania's national roads. Geogaphia. Technica, 12(2), 20-29.

DOI: 10.21163/GT_2017.122.03. 
Chachoua, P.L. (2014). Le système national de santé 1962 a nos jours. Colloque International Sur Les Politiques de Santé Alger. Algeria : Ministère de la Santé, de la Population et de la Réforme Hospitalière en collaboration avec Ecole Nationale Supérieure en Sciences Politiques.

Chen, M., Thill, J.-C. and Delmelle, E. (2018). iGLASS: An Open Source SDSS for Public School Location-Allocation. GeoComputational Analysis and Modeling of Regional Systems. Switzerland AG: Springer, Cham, pp. 325-353.

Coutinho-Rodrigues, J., Simão, A. and Antunes, C.H. (2011). A GIS-based multicriteria spatial decision support system for planning urban infrastructures. Decision Support Systems, 51(3), 720-726.

Cova, T.J. (1999). GIS in emergency management. Geographical information systems, 2(12), 1999.

Cromley, E.K. and McLafferty, S.L. (2011). GIS and Public Health. 2nd ed. The Guilford Press.

Direction de la Programmation et du Suivi Budgétaires (2017). Monograhie de Batna. Wilaya de Batna.

Direction de santé et de population, Wilaya de Batna. (2017). Rapport Annuale de Santé. Wilaya de Batna.

Environmental Systems Research, I. (2008). Geographic Information Systems Providing the Platform for Comprehensive Emergency Management. Available at: https://www.esri.com/library/whitepapers/pdfs/gis-platform-emergency-management.pdf.

Filali, A. and Kalla, M. (2016). Scenario de gestion des crises en milieu urbain - cas d'El Eulma (Algérie). Romanian Journal of Geography, 60(2), 203-211.

Gunes, A.E. and Kovel, J.P. (2000). Using GIS in emergency management operations. Journal of Urban Planning and Development 126(3), 136-149.

Hasnat, M., Islam, M., Hadiuzzaman, M. (2018). Emergency response during disastrous situation in densely populated urban areas: a GIS based approach. Geographia Technica, 13(2), 74-88. DOI: 10.21163/GT_2018.132.06.

Institute, E.S.R. (2018). What Is ModelBuilder? - Help ArcGIS Desktop. Available at: https://desktop.arcgis.com/en/arcmap/latest/analyze/modelbuilder/what-is-modelbuilder.htm [Accessed: 1 February 2020].

Issam, K.M. and Said, G.M. (2017). Using geomatics for assessing vulnerability to cutaneous leis hmanisais. Application to the wilaya of Batna (Algeria). International Journal of GEOMATE 13(40), 9-15.

Johnson, R. (2000). GIS technology for disasters and emergency management.

Makropoulos, C., Butler, D. and Maksimovic, C. (2003). Fuzzy logic spatial decision support system for urban water management. Journal of Water Resources Planning and Management 129(1), 69-77.

Malczewski, J. (1999). GIS and Multicriteria Decision Analysis. Us: John Wiley \& Sons.

Meika, J. (2010). The Benefits of GIS. Available at: https://www.gislounge.com/the-benefits-of-gis/ [Accessed: 4 August 2019].

Mora, M., Forgionne, G.A. and Gupta, J.N. (2003). Decision Making Support Systems: Achievements, Trends, and Challenges for the New Decade. Hershey, PA: Idea Group Pub.

Nasereddine, A. (2016). The conditions of risk management in health facilities-Case study of public institutions nearby care of Algéria/EPSP. Ru'á Iqtișadīyah 6(10), 283-301. doi: https://doi.org/10.12816/0034060.

Nicoară, P.-S. and Haidu, I. (2014). A GIS based network analysis for the identification of shortest route access to emergency medical facilities. Geographia Technica, 9(2), 60-67.

Oufriha, F.Z. (2006). Les réformes du système de santé en Algérie. In: Oufriha, F. Z. (ed.). De Réforme En Réforme: Un Système de Santé à La Croisée Des Chemins. Algeria: Ed. CREAD, pp. 103-116. 
Protection, civil (2017). Disaster History 1962-2012. Algeria: Protection civile Algeria.

Rogers, P. and others (2011). Development of resilient Australia: enhancing the PPRR approach with anticipation, assessment and registration of risks. Australian Journal of Emergency Management, 26(1),54.

Ruda, A. (2014). Spatial decision making for logistics centre allocation. Geographia Technica 9(2), 85-97.

Schneider, P.J. and Schauer, B.A. (2006). HAZUS—its development and its future. Natural Hazards Review, 7(2), 40-44.

Sugumaran, R. and Degroote, J. (2010). Spatial Decision Support Systems: Principles and Practices. Crc Press.

Sultani, R., Soliman, A. and Al-Hagla, K. (2009). The Use of Geographic Information System (GIS) Based Spatial Decision Support System (SDSS) in Developing the Urban Planning Process. Architecture \& Planning Journal, 20, 97-115.

Wangdi, K., Banwell, C., Gatton, M.L., Kelly, G.C., Namgay, R. and Clements, A.C. (2016). Development and evaluation of a spatial decision support system for malaria elimination in Bhutan. Malaria journal 15(1),180.

Zhang, Z., Liu, Y., Li, J. and Chen, B. (2009). Application of GIS and spatial decision support system for affordable housing. Proceedings of 2009 4th International Conference on Computer Science and Education, ICCSE 2009. pp. 1110-1115.

CommunityViz City Explained, Inc. (2003). Available at: https://communityviz.cityexplained.com/communityviz/index.html [Accessed: 1 February 2020].

Hazus | FEMA.Gov. Available at: https://www.fema.gov/hazus [Accessed: 1 February 2020]. 